\title{
Confrontment and solution to gonadotropin resistance and low oocyte retrieval in in vitro fertilization for type I BPES: a case series with review of literature
}

\author{
Yiqi Yu ${ }^{1}$, Mengxia $\mathrm{Ji}^{1}$, Weihai $X \mathrm{u}^{1}$, Ling Zhang ${ }^{1}$, Ming Qi ${ }^{2}$ and Jing Shu ${ }^{{ }^{*}}$ (1)
}

\begin{abstract}
Background: FOXL2 mutations in human cause Blepharophimosis, ptosis, and epicanthus inversus syndrome (BPES). While type II BPES solely features eyelid abnormality, type I BPES involves not only eyelid but also ovary, leading to primary ovarian insufficiency (POI) and female infertility. Current mainstream reproductive option for type I BPES is embryo or oocyte donation. Attempts on assisted reproductive technology (ART) aiming biological parenthood in this population were sparse and mostly unsuccessful.
\end{abstract}

Case presentation: Two Chinese type I BPES patients with low anti-müllerian hormone (AMH) and elevated follicle stimulating hormone (FSH) presented with primary infertility in their early 30s. Genetic studies confirmed two heterozygous duplication mutations that were never reported previously in East Asian populations. They received in vitro fertilization (IVF) treatment and both exhibited resistance to gonadotropin and difficulty in retrieving oocytes in repeated cycles. Doubled to quadrupled total gonadotropin doses were required to awaken follicular response. Patient 1 delivered a baby girl with the same eyelid phenotype and patient 2 had ongoing live intrauterine pregnancy at the time of manuscript submission.

Conclusions: This is the second reported live birth of biological offspring in type I BPES patients, and first success using IVF techniques. It confirmed that ART is difficult but feasible in type I BPES. It further alerts clinicians and genetic counsellors to type female BPES patients with caution in view of the precious and potentially narrowed reproductive window.

Keywords: "Infertility", "Blepharophimosis, ptosis, and epicanthus inversus syndrome", "In vitro fertilization", "FOXL2", "Gonadotropin resistance"

\section{Background}

Blepharophimosis, ptosis, and epicanthus inversus syndrome (BPES) is a rare genetic disease involving predominantly the eyelid and ovary. The prevalence of this disease

\footnotetext{
*Correspondence: shujing@hmc.edu.cn

${ }^{1}$ Department of Reproductive Endocrinology, Reproductive Medicine

Center, Zhejiang Provincial People's Hospital, Affiliated People's Hospital, Hangzhou Medical College, Hangzhou, China

Full list of author information is available at the end of the article
}

is estimated to be 1 in 50,000 births [1]. Interestingly, this pleiotropic effect results from a single gene mutation of FOXL2 rather than from contiguous gene syndrome. 67\% to over $80 \%$ patients were detected mutation-positive in FOXL2 gene after clinical diagnosis based upon four typical eyelid features: blepharophimosis, ptosis, epicanthus inversus, and telecanthus $[2,3]$. Patients with only eyelid features were classified into type II BPES, which was reported to almost always inherit in autosomal dominant pattern, with nearly complete penetrance. Description of original author(s) and the source, provide a link to the Creative Commons licence, and indicate if changes were made. The images or other third party material in this article are included in the article's Creative Commons licence, unless indicated otherwise in a credit line to the material. If material is not included in the article's Creative Commons licence and your intended use is not permitted by statutory regulation or exceeds the permitted use, you will need to obtain permission directly from the copyright holder. To view a copy of this licence, visit http://creativecommons.org/licenses/by/4.0/. The Creative Commons Public Domain Dedication waiver (http://creativeco mmons.org/publicdomain/zero/1.0/) applies to the data made available in this article, unless otherwise stated in a credit line to the data. 
the association of POI (Primary ovarian insufficiency)related female infertility with eyelid features dates back to as early as 1983, almost two decades before the precise gene localization [4-6]. Patients with such phenotypic combination were classified into type I BPES.

Efforts are being made to specify the phenotypegenotype correlation in type I BPES, in order to predict ovarian risk and guide long-term follow-up strategy for affected females at an early age. Unfortunately, unlike the eyelid feature, variable interfamilial and intrafamilial expressivity of ovarian function and fertility was observed [7]. The onset of POI in an individual is neither confidently predictable nor therapeutically reversible at the moment. Current strategy for confirmed type I BPES primarily compromises of two stems: 1) gynecological management of POI by hormone replacement therapy (HRT); 2) Assistance in completing family. The mainstream options for the latter provided at genetic counseling renders biological parenthood, which includes donation of eggs or embryos, adoption or fostering. Precautionary ovarian tissue cryopreservation or oocyte cryopreservation for girls or unmarried women is neither widely used nor reported to be successful for type I BPES yet.

Here we describe two Chinese type I BPES patients with heterozygous FOXL2 duplication mutations that were published for the first time in East Asian populations. The duplication in patient 2 has not been reported to have caused type I BPES previously. More importantly, it was the first reported attempt of IVF on type I BPES patients, which revealed characteristic profile including discordance between antral follicle count (AFC) and anti-müllerian hormone (AMH), gonadotropin resistance, low follicular output rate (FORT) as well as low oocyte retrieval rate (ORR). By sharing our challenge and success, we wish to provide heads-up, hope and help to BPES patients.

\section{Case presentation Clinical history}

Both patients had typical eyelid features and had received plastic surgery in childhood. They presented to our clinic at the age of 32 for infertility. Patient 1 had menarche at the age of 12 , maintaining overall regular menstrual cycle thereafter, except for one episode of menorrhagia which revealed complex hyperplasia and was treated with Mirena. She had a spontaneous pregnancy when she was 30 after actively trying to conceive for one and half years, but unfortunately miscarried at 7 weeks of gestational age. Patient 2 had menarche at the age of 13, followed by oligomenorrhea ever since. Ovulation induction with gonadotropins failed at the dose up to hMG 150 IU/day.

\section{Infertility work-up}

On clinical examination, both patients exhibited welldeveloped secondary sexual characteristics. Body mass index (BMI), Tanner staging of pubic hair and breasts were summarized in Table 1. Both patients' AMH and basal hormone profile implied decreased ovarian reserve, But ultrasound scan discordantly showed plenty bilateral antral follicles (Table 1). Their uteri were of normal size. For both patients, hysterosalpingogram and husband's semen analysis were unremarkable [7].

\section{IVF treatment}

Antral follicle count (AFC): number of follicles with diameter between 4 milimetre $(\mathrm{mm})$ to $9 \mathrm{~mm}$ seen at day 2-3 of menses.

Preovulatory follicle count (PFC): number of follicles with diameter between $14 \mathrm{~mm}$ to $22 \mathrm{~mm}$.

Follicular output rate $(\mathrm{FORT})=\mathrm{PFC} / \mathrm{AFC} \times 100 \%[8]$.

Oocyte retrieval rate $(\mathrm{ORR})=$ number of oocytes retrieved/PFC $\times 100 \%[9]$.

Both patients received two cycles of controlled ovarian hyperstimulation $(\mathrm{COH})$ and obtained only one embryo. Patient 1 received first cycle in another centre and second cycle in our centre. Details and outcomes of $\mathrm{COH}$ in her first cycle were obtained from her outpatient medical record with authorization. Patient 2 received both cycles in our centre.

In first $\mathrm{COH}$ cycle for patient 1 , unexpected gonadotropin resistance was observed under GnRH agonist protocol, rendering her a long hyperstimulation course with increasing dose of hMG up to a maximum of $450 \mathrm{IU}$ per day. Not one oocyte was harvested from the six preovulatory follicles. GnRH antagonist protocol was used for her second cycle in our centre. $\mathrm{COH}$ was started at a dose of rFSH 150 IU plus hMG 150 IU per day, and was increased to $\mathrm{rFSH} 150 \mathrm{IU}$ plus hMG $300 \mathrm{IU}$ per day at the sixth day of $\mathrm{COH}$ due to poor response (Fig. 1a). In view of previous oocyte retrieval failure, the oocyte retrieval time was postponed to $40 \mathrm{~h}$ post ovulation-triggering instead of the routine $36 \mathrm{~h}$. two preovulatory follicles were punctured, but only one oocyte was harvested on first flushing using double-lumen needle. The FORT and ORR were 0.16 and 0.5 respectively despite doubled total gonadotropin dose, much lower than those of patients at the same age undergoing IVF in our centre (Table 2). This mature oocyte was normally fertilized into 2PN (2pronuclear) zygote following conventional IVF technique, and a blastocyst graded $5 \mathrm{AC}$ was frozen.

In first $\mathrm{COH}$ cycle for patient 2, combined use of hMG and clomiphene citrate was administrated simultaneously at a starting dose of $225 \mathrm{IU} /$ day and $100 \mathrm{mg} /$ day from day 3 of menses. Both ovaries were barely 


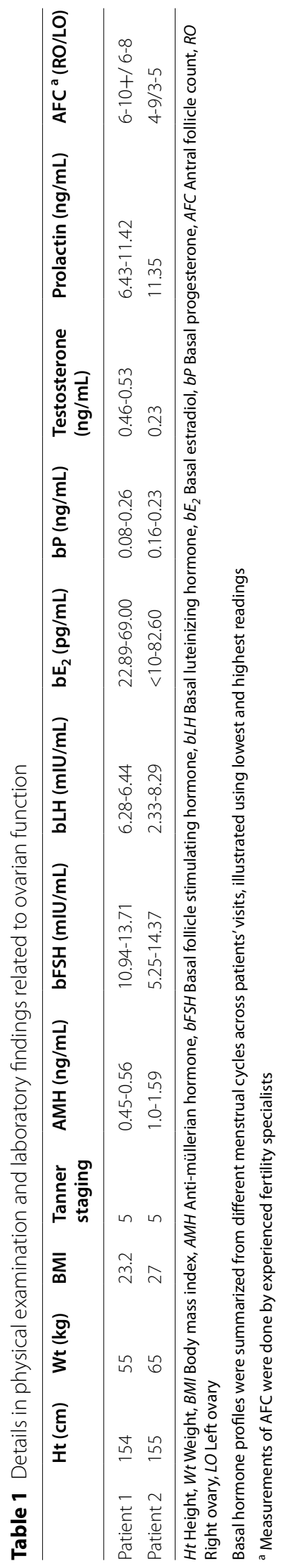



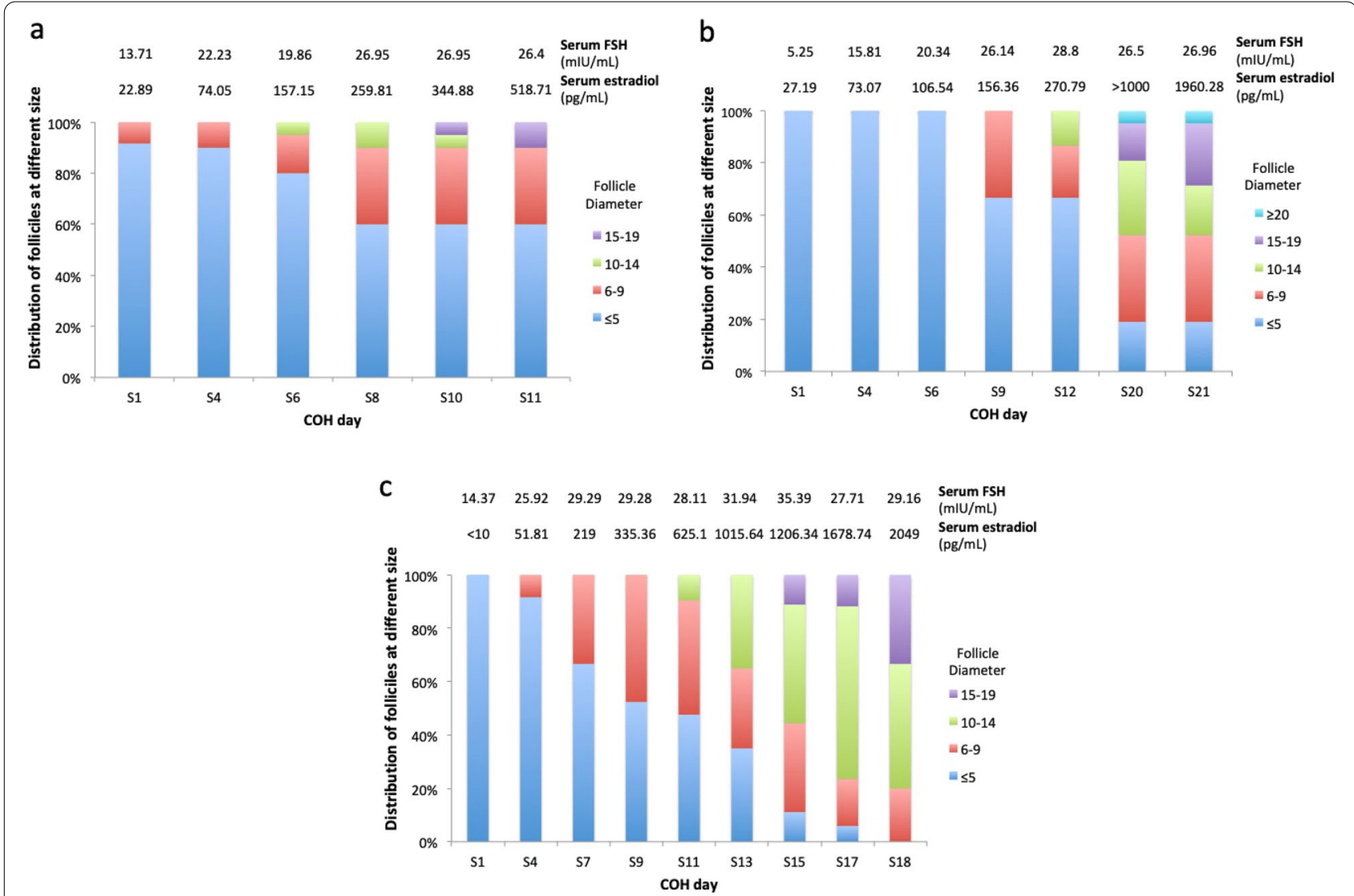

Fig. 1 Follicular growth under controlled ovarian hyperstimulation $(\mathrm{COH})$ showing the relationship between serum FSH level and follicular response as well as the corresponding serum estradiol level. Follicles are divided into 5 groups: $\leq 5 \mathrm{~mm}, 6-9 \mathrm{~mm}, 10-14 \mathrm{~mm}, 15-19 \mathrm{~mm}, \geq 20 \mathrm{~mm}$. Percentage of each group is shown. S1 = Day 1 of hyperstimulation. a Patient 1's second COH cycle; b Patient 2's first COH cycle, note that no exact estradiol reading was recorded on S2O because the corresponding serum sample was not diluted; c Patient 2's second COH cycle

responsive to serum $\mathrm{FSH}$ at $20 \mathrm{mIU} / \mathrm{mL}$ achieved by hMG stepped-up to $450 \mathrm{IU} /$ day. HMG was eventually increased to $525 \mathrm{IU} /$ day, and serum FSH reached 28.8 $\mathrm{mIU} / \mathrm{mL}$ (Fig. 1b). After a total of 21 days of hyper-stimulation with a cumulative dose of gonadotropin at $9600 \mathrm{IU}$ and clomiphene citrate at $2100 \mathrm{mg}$, seven preovulatory follicles developed but only one oocyte was harvested. This mature oocyte was normally fertilized following conventional IVF technique, but embryo development unfortunately arrested on day 2 . A similar protocol was used in her second cycle, taking up 18 days and a cumulative dose of hMG at $8850 \mathrm{IU}$ plus clomiphene citrate at $2300 \mathrm{mg}$. simultaneous use of hMG at $450 \mathrm{IU} /$ day plus clomiphene citrate at $100 \mathrm{mg} /$ day was started from day 3 of menses. HMG was increased to $525 \mathrm{IU} /$ day and clomiphene citrate increased to $150 \mathrm{mg} /$ day at $\mathrm{COH}$ day 9 due to poor response. Resultant serum FSH ranged from 25 to $35 \mathrm{mIU} / \mathrm{mL}$ (Fig. 1c). Three oocytes were harvested from seven preovulatory follicles, and were all mature. After conventional IVF, one 2PN zygote developed into a Grade-2 7-cell Cleavage-stage embryo with 5\% fragmentation. The rest two oocytes ended up unfertilized (OPN) and abnormally fertilized (3PN) respectively. For both cycles the ovulation triggering-to-harvesting interval was $36 \mathrm{~h}$, and oocytes were retrieved using double lumen needles on one to three times of flushing. The FORT and ORR were low, but improvement was seen in second cycle (Table 2).

\section{Genetic analysis}

For each participant, genomic DNA was extracted from whole blood samples. High throughput target region sequencing (TRS) was carried out to cover all exons and $\pm 10 \mathrm{bp}$ of neighboring introns, with depth at $122.15 \times$ and average coverage at $99.36 \%$. All candidate variants were annotated and filtered against public databases including 1000 Genome, gnomAD, ClinVar, dbNSFP, LOVD and ZJU-DB. PCR and Sanger sequencing was performed to confirm variants within patients and other members in the family. In-silico analysis with the online tool PROVEAN was implemented. Mutation nomenclature was made according to Human Genome 
Variation Society (HGVS). Integrated interpretation was made according to American College of Medical Genetics and Genomics (ACMG) guideline.

For patient 1, genetic testing revealed a de novo FOXL2 duplication mutation (c.843_859dupGGCCGC ACCCCCGCCTC) resulting in frameshift and premature termination (Fig. 2, Additional file 1). For patient 2, genetic testing uncovered a FOXL2 duplication mutation (c.178_192dupGGCGATGAGCGCCAC) resulting in insertion of five hydrophobic amino acids, Val-Ala-LeuIle-Ala, between the 64th and 65th amino acids (Fig. 2, Additional file 1). It was likely a de novo mutation in view that neither eyelid abnormality nor infertility was reported in her family (Fig. 3b). Both couples opted not for preimplantation genetic testing (PGT) after thorough genetic counseling.

\section{Pregnancy outcomes}

The frozen-thawed blastocyst of patient 1 was transferred after hormonal replacement preparation of endometrium. Pregnancy was confirmed on Day 9 post embryo transfer, and carried on uneventfully until 33weeks of gestational age, when patient 1 was hospitalized for antepartum bleeding and shortened cervix. She delivered a baby girl at 36 weeks by caesarean section after prolonged preterm premature rupture of membrane (PPROM). The baby weighed $2600 \mathrm{~g}$ at birth, and manifested the same eyelid features as her mother (picture see "Additional file 2").

The frozen-thawed Cleavage-stage embryo of patient 2 was transferred after hormonal replacement preparation of endometrium. Pregnancy was confirmed on Day 9 post embryo transfer, and latest follow-up at the time of manuscript submission confirmed ongoing single live pregnancy at 29 weeks of gestation.

\section{Literature search}

We searched the PubMed database using search terms for Medical Subject Headings and/or text words relating to FOXL2 or BPES. The retrieved articles were further hand-searched using our inclusion criteria (papers reporting pregnancy in type I BPES). Only 1 case of type I BPES was reported to have achieved live birth by AID with monofollicular ovulation induced by high dose of rFSH (Table 3). No natural pregnancy was reported.

\section{Discussion}

FOXL2 gene belongs to the winged-helix/forkhead transcription factor family, members of which are highly conservative. Pathogenic variants lead to either disordered subcellular distribution or dysregulation of downstream genes [10]. Apart from the eyelid, FOXL2 is most abundantly expressed in the ovary $[11,12]$. It is an earliest granulosa cell marker, and plays a crucial role in sex determination, tumorigenesis, ovarian somatic cell proliferation and differentiation [13].

Both of the two patients presented to our reproductive medicine clinic for infertility with underlying ovarian function defect. Since their eyelid abnormality occurred early at birth and were surgically corrected before good understanding of the relationship between FOXL2 mutation and BPES [4], in addition to the absence of family history, genetic diagnosis was delayed until adulthood.

During their fertility treatment, they were clinically resistant to gonadotropins, but not completely unresponsive. Furthermore, both exhibited paradoxically normal AFC despite low AMH, and thus did not fall into any of the four groups under Poseidon criteria [14]. Beyond that, both experienced poor ORR in repeated IVF cycles despite multiple flushing [15], conform to poor ovarian responders according to Bologna criteria [16]. In spite of all these difficulties, they were lucky to have succeeded with their one and only embryo.

This is inspiring news considering the very limited successes reported in biological parenthood achieved by assisted reproductive technology (ART) for BPES patients (Table 3). In a large cohort of 164 probands, a 31-year-old BPES patient (c.672_701dup) without hypergonadotropic hypogonadism was mentioned to have achieved pregnancy after IVF treatment, but indication for IVF was not specified [17]. A case report in 2014 described a 30-year-old nulligravid woman with clinically diagnosed type I BPES, who delivered twins after artificial insemination with donor sperm (AID) [18]. Similar ovarian resistance as our patients was observed during her gonadotropin stimulation, and follicles were unresponsive to $\mathrm{rFSH}$ at $125 \mathrm{IU} /$ day. Monofollicular ovulation was achieved by 14-day course of rFSH at $300 \mathrm{IU} /$ day. Although genetic evidence was not provided in that case report, typical eyelid changes in one twin seemed to support the presence of genetic inheritance, probably FOXL2 mutation [3].

In patient 1 (c.843_859dup), insertion of 17 nucleotides (Fig. 2) resulted in frameshift in open reading frame (ORF) and a protein truncated after polyalanine tract (PAT), which belonged to group D according to classification suggested by De Baere E et al. [19]. It was a mutational hotspot responsible for approximately 13-18\% of all intragenic pathogenic variants [17, 20], but was reported for the first time here in East Asian populations. Both type II BPES phenotypes with naturally completed family and type I BPES phenotypes had been observed in pedigrees with this mutational variant, revealing phenotypic variability and even variable intrafamilial expressivity of POI [17]. 


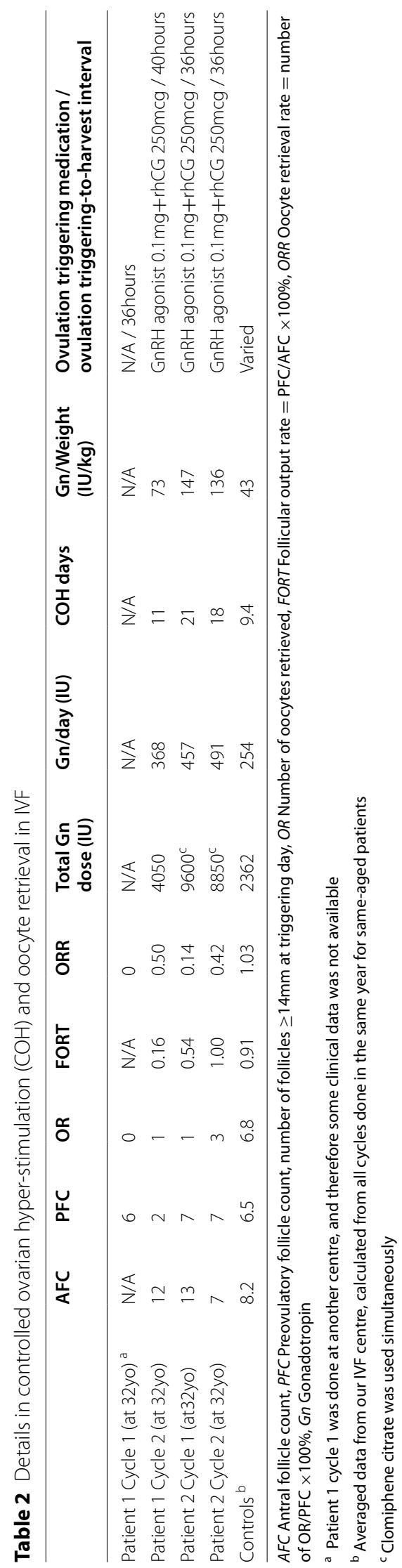




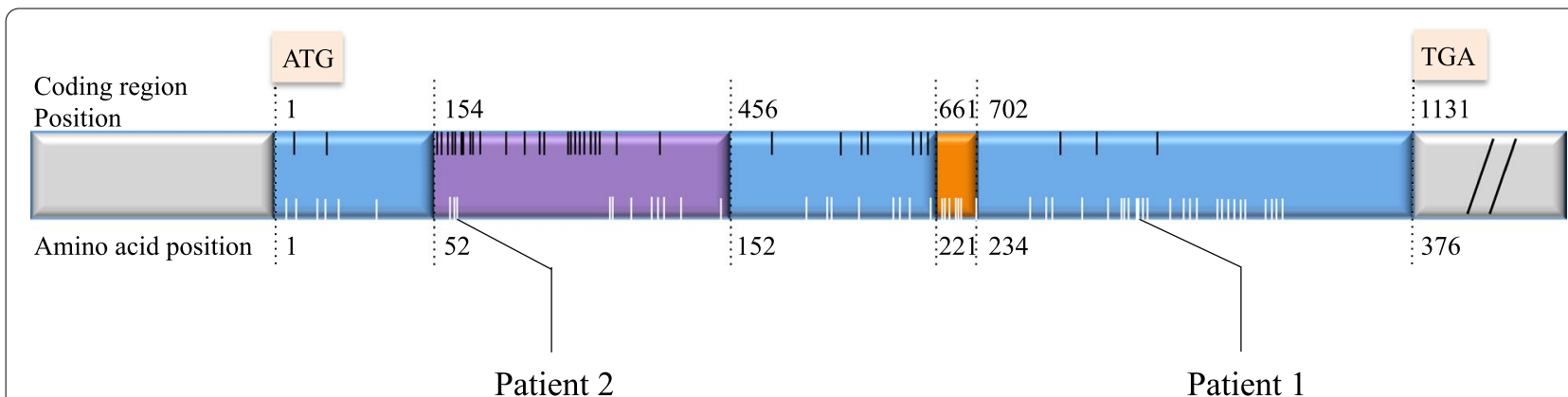

Fig. 2 Positions of mutations detected in the two patients. Purple region: forkhead domain (FHD); Orange region: polyalanine tract (PAT); Blue region: the rest of coding region. Pathogenic or likely-pathogenic mutations submitted in ClinVar were summarized, presenting as short vertical lines: black line = point mutation; white line = mutation resulting in deletion or duplication

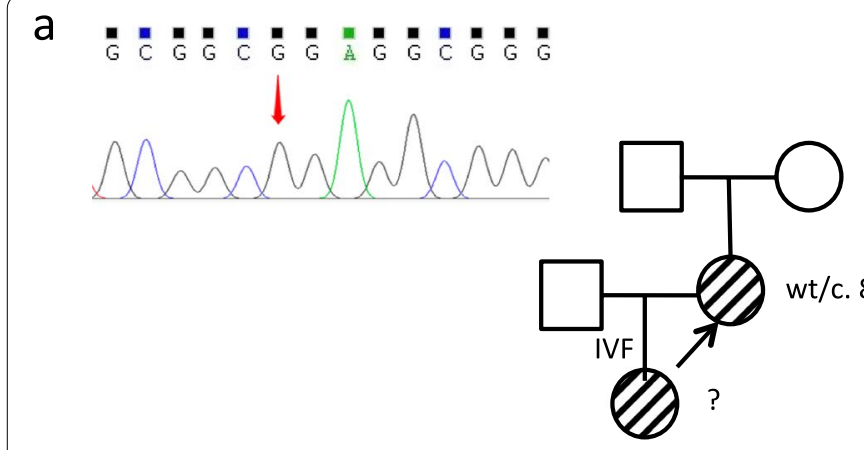

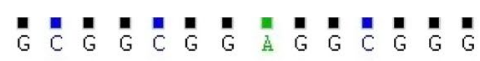

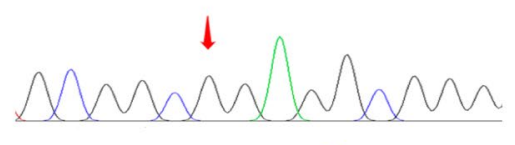

$\dot{G} \bar{G} \bar{C} \bar{C} \bar{G} \overline{\mathbb{A}} \bar{C} \bar{G} \bar{G} \bar{G} \bar{C} \bar{C}$

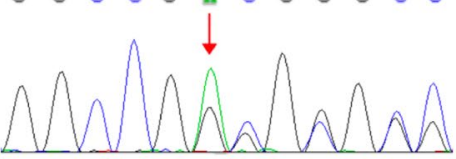

b

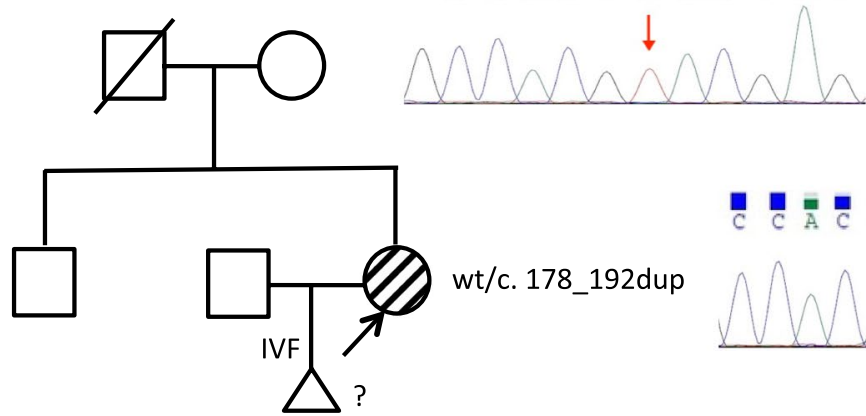

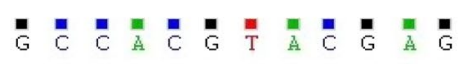

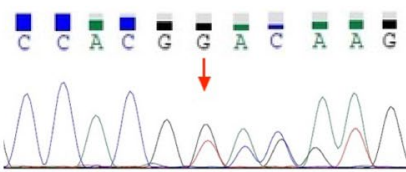

Fig. 3 Pedigrees and Sanger sequencing of patients and their relatives. a Sanger sequencing for patient 1 and her parents is demonstrated. $\mathbf{b}$ Sanger sequencing for patient 2 and her mother is demonstrated

The rarer c.178_192 duplication found in patient 2 leads to an elongated protein. Noteworthily, the insertion of five hydrophobic amino acids occurs in the regulatory forkhead domain (FHD) (Fig. 2). Studies have shown that missense mutations in the FHD impaired not only subcellular localization but also transactivation activity of FOXL2, with either loss-of-function or dominant- negative effect [21]. FHD-deleted FOXL2 mutants lost the direct binding ability to SF-1 in vitro, which mediated its repression on steroidogenic genes CYP17 [22]. Elongation from inside the FHD was not included in the classification suggested by De Baere E et a l. [19], but considering that it occurred immediately after the start of this functional domain, deranged 


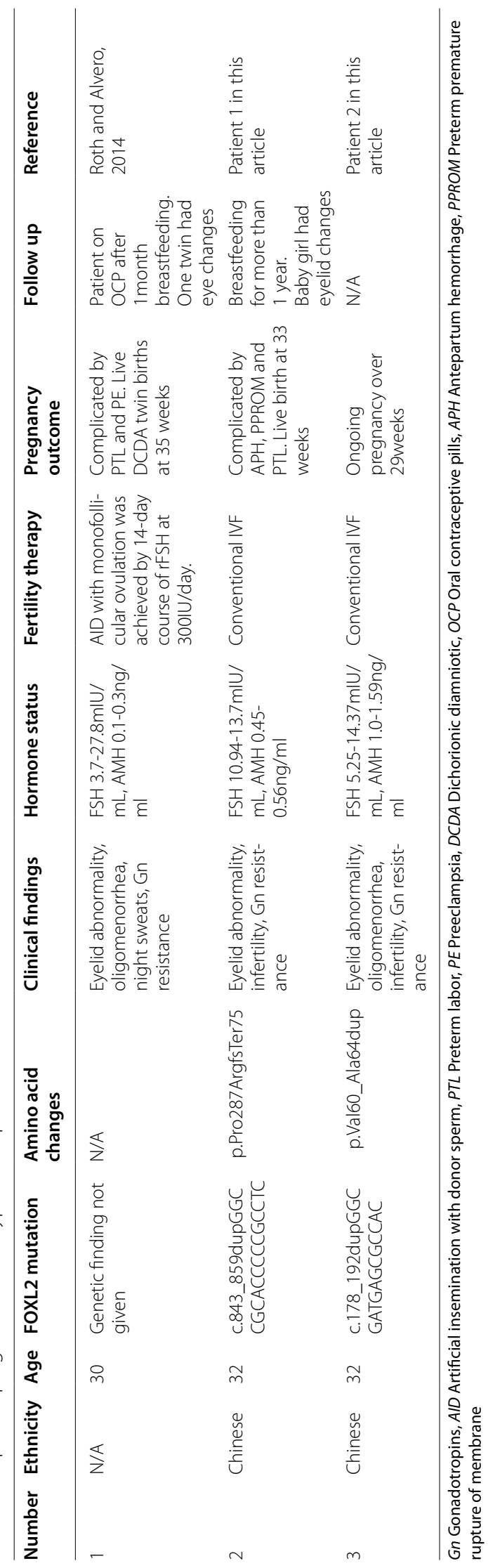


protein function in regulatory activity was suspected. Clinically patient 2 manifested more severe phenotype with oligomenorrhea. Ovarian resistance was also stronger, even after adjustion for body weight. First described pedigree with this duplication was a mother and son with BPES eyelid features. The mother reported oligomenorrhea and menorrhagia. Ovarian function and age of reproduction was not mentioned. Since there was absence of POI and infertility history, the mother was grouped into type II BPES. Second case was a British boy aged 8 in 2019 [2], but family history was not stated. To our knowledge, this mutation was never reported in East Asian populations, and never reported to have caused classic type I BPES phenotype.

Further studies are needed to elucidate the pathophysiology of the distinctive ovarian characteristics in these FOXL2 mutants:

1) Actual follicular reserve behind the discordance between AFC and AMH: Considering the relative abundance of gonadotropin-responsive (even though resistant) antral follicles in both of our patients at their age, the mechanism of low AMH in type I BPES patients may differ from patients with non-genetic causes of ovarian insufficiency, such as advanced age, or acquired pathology (e.g endometriosis, surgery

, or chemotherapy) [23]. AMH is produced in primordial follicles, preantral follicles and small antral follicles [24]. It works as an inhibitor of upstream primordial follicle recruitment [25]. FOXL2 works within a broader span of time along the follicular development, from multipotential stromal cells to cumulus cells in preovulatory follicles [13]. It was speculated that the mutual regulation of the two genes formed a positive feedback loop to maintain ovarian reserve [26]. Small follicles expressing mutant FOXL2 may have lowered expression of $A M H$, leading to the paradoxy.

Without the guarding by properly-expressed $A M H$ and FOXL2 at different stages, is there imminently exhausted primordial follicle pool behind the ultrasonographic normal AFC? We do not have ovarian tissue histology to uncover the real situation in our two patients, but previous studies suggested risk of follicular depletion at an alarming rate, which eventually led to the well-known final stage of POI in type I BPES. Two siblings with a strong family history of autosomal dominant inheritance of BPES were reported in 1988 , before establishment of genetic causation. The elder sister aged 28 and the younger sister aged 20 presented with secondary amenorrhea and hypergonadotropic hypogonadism. Ovarian pathological sections obtained from laparoscopy respectively showed true premature menopause with total absence of primordial follicles in the elder sister, and normal number of primordial follicles in the younger sister [27]. In Foxl2 $2^{\text {lac } Z}$ homozygous murine ovaries, the presence of defected squamous-to-cuboidal transition and premature expression of Gdf9 indicated accelerated depletion of primordial follicles and oocytes atresia, providing plausible explanation for the elder sister's histological finding. In another paper, two patients with FOXL2 deletions after PAT and resultant putative elongated proteins were reported. The 19 -year-old patient suffered from primary amenorrhea with underlying low AMH and abnormal ovarian histology (polyovular follicles, defective basal lamina, deposition of cholesterol crystals, advanced expression of proliferative marker Ki67, and intracytoplasmic FOXL2 aggregation). The 25-year-old patient presented with infertility, but had normal AMH and normal ovarian reserve clinically, ultrasonographically, and histologically. But there was also evidence of uncommon advanced expression of Ki67 in transitional follicles implying accelerated recruitment [28].

2) Gonadotropin resistance: Our patients were resistant to gonadotropins, yet responsive at high doses with extended courses. Target serum FSH seemed to be higher than 25 to $30 \mathrm{mIU} / \mathrm{mL}$. Doubled to quadrupled total gonadotropin doses in comparison to general population was required in various $\mathrm{COH}$ protocols in order to awaken follicular response, and clomiphene citrate was added to patient 2 for enhancement.

Clomiphene citrate is a selective estrogen receptor modulator. It was utilized in ovulation induction or stimulation by preventing estrogen negative feedback and thereby stimulating endogenous gonadotropin release. It was shown that supplement of clomiphene improved ovarian stimulation outcomes in IVF treatment and decreased total gonadotropin dosage, which applied to women with advanced age and/or poor response [29, 30]. We adopted the combined use of clomiphene citrate and gonadotropin into the $\mathrm{COH}$ treatment for our two type I BPES patients. Both of the patients were on the road of accelerated ovarian function decline in addition to gonadotropin resistance. However, unlike patients with POI or imminent POI due to advance age or iatrogenic cause, supraphysiological level of serum FSH level seemed to correlate positively to ovarian response and IVF outcome.

As a result of the augmentation of hyperstimulation, FORTs could be improved but still low compared to general IVF patients. Apart from the AID case mentioned above, who succeeded by using high dose $\mathrm{rFSH}$, few case 
reports, before and after FOXL2 gene localization, also described the phenomenon of ovarian resistance to gonadotropins in BPES patients [27, 31-34], but failed either in ovulation induction or in conception. Is the resistance universal to type I BPES patients? Is it the major cause of infertility? Answers are not clear yet. We speculate that the genes involved in FSH responsiveness and/or antral follicle growth, such as FSH receptor (FSHR), might be dysregulated in mutants through direct or indirect mechanisms.

3) Abnormal ovulation and low ORR: Less studies focused on ovulation in BPES patients. As described, patient 2 was oligomenorrheic secondary to anovulation. Patient 1 maintained overall natural normal cycle with sonographically confirmed ovulation. It was at least once followed by successful natural fertilization and implantation but unluckily ended up with first-trimester miscarriage. However, she also reported to have experienced cycles judged as luteinized unruptured follicle syndrome (LUFS). Furthermore, the history of endometrial hyperplasia implied possible preceding anovulation. There might be some mechanism behind the disordered ovulation of graafian (preovulatory) follicles as well as the difficulty in harvesting oocytes from them in IVF. Hypotheses include hampered oocyte maturation, abnormal expression of Luteinizing hormone receptor (LHR) [35], defected gap junction, and deranged activity of local factors such as prostaglandin and proteases [36]. Individualized ovulation triggering-to-harvesting interval and two-chamber needle flushing may have a role in improving the ORR. In view of the likely innate gonadotropin resistance, an increased dose of LH/hCG dosage for triggering, which was not tried in our cases, may also contribute potential benefit.

Currently mainstream recommendation at genetic counseling for type I BPES patients includes donation of eggs or embryos, adoption or fostering, sacrificing biological parenthood. This is probably based on the following considerations: 1 ) missed reproductive window at presentation; 2) limited translation of in vitro maturation (IVM) into clinical use; 3) ethical concerns about precautionary ovarian tissue cryopreservation or oocyte cryopreservation for young girls with unclear ovarian phenotype; 4) risk of inheritance of pathogenic mutations.

Noteworthily on the other hand, patients manifesting either type of BPES usually have normal menarche and secondary sex characteristics. Association with major defects including intellectual disability were rare with inconclusive causation. Even type I BPES phenotypes with primary or secondary amenorrhea possess potential capacity of natural conception and deserve a childbearing opportunity. Early intervention may rescue their threatened fertility.

Clinicians and scientists were making efforts to specify the phenotype-genotype correlation in ovarian function [19]. Studies suggested high POI risk from mutations with proteins truncated upstream of PAT and from mutations involving inward-pointing of relevant amino acids towards the hydrophobic core. Meanwhile PAT expansion predominantly leads to type II BPES [10]. However, considering the inter-individual variability with even varied intrafamilial penetrance of ovarian phenotypes from identical genotypes, POI risk should be emphasized to all female patients as early as possible while an efficient prediction model is still on the way. As the putative acceleration of follicular depletion takes place without clear red flag for onset and exhaustion, it is unrational to declare innocence and passively wait till the age of 40 to diagnose POI, especially considering the much higher difficulty they might encounter like our patients, compared to patients with other causes of low ovarian reserve.

In summary, we described detailed IVF treatment to two Chinese type I BPES patients, whose FOXL2 mutations were new to East Asian population database. Their ovarian profiles seem to differentiate type I BEPS from either pure resistant ovarian syndrome (ROS) or pure POI, and offers materials for further basic research on FOXL2 gene and type I BPES. Of course, due to the rarity of the disease, we look forward to more cases to reproduce the success and the observed phenomena. Nevertheless, our report confirmed that IVF in type I BPES is difficult, but still feasible with individualized treatment. It also serves as a stepping-stone to future attempts on IVM and PGT in this population.

\section{Abbreviations}

AFC: Antral follicle count; AID: Artificial insemination with donor sperm; AMH: Anti-müllerian hormone; ART: Assisted reproductive technology; BPES: Blepharophimosis, ptosis, and epicanthus inversus syndrome; $\mathrm{COH}$ : Controlled ovarian hyperstimulation; FORT: Follicular output rate; FSH: Follicle stimulating hormone; GnRH: Gonadotropin-releasing hormone; hMG: Human menopausal gonadotropin; IVF: In vitro fertilization; LH: Luteinizing Hormone; ORR: Oocyte retrieval rate; PGT: Preimplantation genetic testing; POI: Primary ovarian insufficiency; PN: Pronuclear; ROS: Resistant ovarian syndrome.

\section{Supplementary Information}

The online version contains supplementary material available at https://doi. org/10.1186/s13048-021-00900-2.

Additional file 1. Summary of genetic analysis findings (table).

Additional file 2. Patient 1's daughter-facial manifestations. 


\section{Acknowledgements}

We are grateful to Dr. Yi Zhai (University of Alberta) and Dr. He Jinjing (Department of Ophthalmology, Zhejiang Provincial People's Hospital) who provided professional help to this work from ophthalmological perspective.

\section{Authors' contributions}

YYQ was responsible for the diagnosis, clinical management and manuscript drafting. JMX helped in the diagnosis and clinical management of the two patients. ZL and XWH were responsible for laboratory procedures in IVF. QM contributed to genetic testing and interpretation, as well as manuscript revising. SJ supervised the clinical management and revised the manuscript. All authors have read and approved the final manuscript

\section{Authors' information}

Yu Yiqi (Master of Medicine, MRCOG): Obstetrician and Gynaecologist, Department of Reproductive Endocrinology, Reproductive Medicine Center, Zhejiang Provincial People's Hospital, Affiliated People's Hospital of Hangzhou Medical College, Hangzhou, China.

\section{Funding}

The study was supported by General Research Program for Medicine and Health of Zhejiang Province (2019KY033 to JS, 2020KY414 to JS).

\section{Availability of data and materials}

The datasets supporting the conclusions of this article are included within the article (and its supplementary files).

\section{Declarations}

\section{Consent for publication}

Written informed consents for publication of clinical details and/or clinical images were obtained from the patients/patient's guardians.

\section{Competing interests}

The authors declare that there is no conflict of interest that could be perceived as prejudicing the impartiality of this article.

\section{Author details}

'Department of Reproductive Endocrinology, Reproductive Medicine Center Zhejiang Provincial People's Hospital, Affiliated People's Hospital, Hangzhou Medical College, Hangzhou, China. ${ }^{2}$ Department of Cell Biology and Medical Genetics, Zhejiang University School of Medicine, Hangzhou, China.

Received: 5 Auqust 2021 Accepted: 15 October 2021

Published online: 28 October 2021

\section{References}

1. Oley C, Baraitser M. Blepharophimosis, ptosis, epicanthus inversus syndrome (BPES syndrome). J Med Genet. 1988;25:47-51.

2. Bunyan DJ, Thomas NS. Screening of a large cohort of blepharophimosis, ptosis, and epicanthus inversus syndrome patients reveals a very strong paternal inheritance bias and a wide spectrum of novel FOXL2 mutations. Eur J Med Genet. 2019;62:103668.

3. D'haene B, Nevado J, Pugeat M, et al. FOXL2 copy number changes in the molecular pathogenesis of BPES: unique cohort of 17 deletions. Hum Mutat. 2010;31:E1332-47.

4. de Baere E, Dixon MJ, Small KW, et al. Spectrum of FOXL2 gene mutations in blepharophimosis-ptosis-epicanthus inversus (BPES) families demonstrates a genotype--phenotype correlation. Hum Mol Genet. 2001;10:1591-600.

5. Zlotogora J, Sagi M, Cohen T. The blepharophimosis, ptosis, and epicanthus inversus syndrome: delineation of two types. Am J Hum Genet. 1983;35:1020-7.

6. Cocquet J, Pailhoux E, Jaubert F, et al. Evolution and expression of FOXL2. J Med Genet. 2002;39:916-21.

7. Cooper TG, Noonan E, von Eckardstein S, et al. World Health Organization reference values for human semen characteristics. Hum Reprod Update. 2010;16:231-45
8. Gallot V, Da Berwanger Silva AL, Genro V, Grynberg M, Frydman N, Fanchin R. Antral follicle responsiveness to follicle-stimulating hormone administration assessed by the follicular output RaTe (FORT) may predict in vitro fertilization-embryo transfer outcome. Hum Reprod. 2012:27:1066-72

9. Younis JS, Skournik A, Radin O, Haddad S, Bar-Ami S, Ben-Ami M. Poor oocyte retrieval is a manifestation of low ovarian reserve. Fertil Steril. 2005;83:504-7

10. Elzaiat $M$, Todeschini A-L, Caburet $S$, Veitia RA. The genetic make-up of ovarian development and function: the focus on the transcription factor FOXL2. Clin Genet. 2017:91:173-82.

11. Huang $\mathrm{S}$, Ye L, Chen H. Sex determination and maintenance: the role of DMRT1 and FOXL2. Asian J Androl. 2017;19:619-24.

12. Duffin K, Bayne RAL, Childs AJ, Collins C, Anderson RA. The forkhead transcription factor FOXL2 is expressed in somatic cells of the human ovary prior to follicle formation. Mol Hum Reprod. 2009;15:771-7.

13. Pisarska MD, Bae J, Klein C, Hsueh AJW. Forkhead 12 is expressed in the ovary and represses the promoter activity of the steroidogenic acute regulatory gene. Endocrinology. 2004;145:3424-33.

14. Humaidan P, Alviggi C, Fischer R, Esteves SC. The novel POSEIDON stratification of 'Low prognosis patients in Assisted Reproductive Technology' and its proposed marker of successful outcome. F1000Res. 2016:5:2911.

15. Xiao Y, Wang Y, Wang M, Liu K. Follicular flushing increases the number of oocytes retrieved in poor ovarian responders undergoing in vitro fertilization: a retrospective cohort study. BMC Womens Health. 2018;18:186.

16. Ferraretti AP, La Marca A, Fauser BCJM, Tarlatzis B, Nargund G, Gianaroli L. ESHRE consensus on the definition of 'poor response' to ovarian stimulation for in vitro fertilization: the Bologna criteria. Hum Reprod. 2011:26:1616-24.

17. Beysen D, de Jaegere $S, A$ mor D, et al. Identification of 34 novel and 56 known FOXL2 mutations in patients with Blepharophimosis syndrome. Hum Mutat. 2008;29:E205-19.

18. Roth LW, Alvero R. Pregnancy in a woman with premature ovarian insufficiency associated with blepharophimosis, ptosis, epicanthus inversus syndrome type I. a case report. J Reprod Med. 2014;59:87-9.

19. de Baere E, Beysen D, Oley C, et al. FOXL2 and BPES: mutational hotspots, phenotypic variability, and revision of the genotype-phenotype correlation. Am J Hum Genet. 2003;72:478-87.

20. Beysen D, de Paepe A, de Baere E. FOXL2 mutations and genomic rearrangements in BPES. Hum Mutat. 2009:30:158-69.

21. Beysen D, Moumné L, Veitia R, et al. Missense mutations in the forkhead domain of FOXL2 lead to subcellular mislocalization, protein aggregation and impaired transactivation. Hum Mol Genet. 2008;17:2030-8.

22. Park M, Shin E, Won M, et al. FOXL2 interacts with steroidogenic factor-1 (SF-1) and represses SF-1-induced CYP17 transcription in granulosa cells. Mol Endocrinol. 2010;24:1024-36.

23. Jin $M, Y u Y$, Huang $H$. An update on primary ovarian insufficiency. Sci China Life Sci. 2012;55:677-86.

24. Benayoun BA, Batista F, Auer J, et al. Positive and negative feedback regulates the transcription factor FOXL2 in response to cell stress: evidence for a regulatory imbalance induced by disease-causing mutations. Hum Mol Genet. 2009;18:632-44.

25. Schmidt D, Ovitt CE, Anlag K, et al. The murine winged-helix transcription factor Fox 2 is required for granulosa cell differentiation and ovary maintenance. Development. 2004;131:933-42.

26. Thanatsis N, Kaponis A, Koika V, Georgopoulos NA, Decavalas GO. Reduced Foxo3a, FoxL2, and p27 mRNA expression in human ovarian tissue in premature ovarian insufficiency. Hormones (Athens). 2019;18:409-15.

27. Fraser IS, Shearman RP, Smith A, Russell P. An association among blepharophimosis, resistant ovary syndrome, and true premature menopause. Fertil Steril. 1988:50:747-51.

28. Méduri G, Bachelot A, Duflos C, et al. FOXL2 mutations lead to different ovarian phenotypes in BPES patients: case report. Hum Reprod. 2010;25:235-43.

29. Triantafyllidou O, Sigalos G, Gkoles L, et al. The addition of clomiphene citrate to ovarian stimulation protocols for poor responders. Eur J Obstet Gynecol Reprod Biol. 2020;251:136-40.

30. Liang Y, Guo Q, Wu X-H, et al. Does the additional use of clomiphene citrate or letrozole for in vitro fertilization deserve more attention? BMC Pregnancy Childbirth. 2021;21:275. 
31. Graf M, Distler W, Schnürch HG, Majewski F. Ovarialinsuffizienz bei Blepharophimose, ptosis, epicanthus inversus. Geburtshilfe Frauenheilkd. 1986;46:187-9.

32. Panidis D, Rousso D, Vavilis D, Skiadopoulos S, Kalogeropoulos A. Familial blepharophimosis with ovarian dysfunction. Hum Reprod. 1994;9:2034-7.

33. Morales M, Chardonnens D, Bottani A, Gersbach-Forrer M, Campana A. Le syndrome du blépharophimosis-ptosis-epicanthus inversus associé à une stérilité. J Gynecol Obstet Biol Reprod (Paris). 1999;28:833-7.

34. Siewert AL, Stein Q, Flanagan J, Hansen KA. Blepharophimosis-ptosisepicanthus inversus syndrome and hypergonadotropic hypogonadism. Fertil Steril. 2016;2008(90):e11-2.

35. Greene AD, Patounakis G, Segars JH. Genetic associations with diminished ovarian reserve: a systematic review of the literature. J Assist Reprod Genet. 2014;31:935-46.

36. Rosewell KL, Al-Alem L, Zakerkish F, et al. Induction of proteinases in the human preovulatory follicle of the menstrual cycle by human chorionic gonadotropin. Fertil Steril. 2015;103:826-33.

\section{Publisher's Note}

Springer Nature remains neutral with regard to jurisdictional claims in published maps and institutional affiliations.

- fast, convenient online submission

- thorough peer review by experienced researchers in your field

- rapid publication on acceptance

- support for research data, including large and complex data types

- gold Open Access which fosters wider collaboration and increased citations

- maximum visibility for your research: over $100 \mathrm{M}$ website views per year

At BMC, research is always in progress.

Learn more biomedcentral.com/submissions 